TAO, Vol. 12, No. 4, 649-662, December 2001

\title{
On Two Methods Using Magnetometer-array Data for Studying Magnetic Pulsations
}

\author{
Peter J. Chi ${ }^{1, *}$ and Christopher T. Russell ${ }^{1}$
}

(Manuscript received 29 December 2000, in final form 9 October 2001)

\begin{abstract}
We present some recent progress in interpreting the results from the two powerful methods that analyze the magnetic pulsations observed by ground magnetometer arrays. The first method involves an inversion technique that requires the observations from a chain of magnetometer stations located on the same latitude. This method can estimate several factors that affect the pulsation amplitude on the ground, namely the magnitude of the wave "event", the local time distribution, and the ground conductivity effect. By analyzing several months of pulsation data, we present an alternative approach to estimate the statistical values of the ground conductivities. We confirm the conductivity structure obtained by one day of data presented by Chi et al. (1996). We also demonstrated the local time dependence of wave amplitude for different frequency bands using this method. The second technique is the "the gradient method" that calculates the difference in phase or amplitude of the signals measured at two closely separated stations on the same meridian. This technique has been successful in "observing" the eigenfrequencies of magnetospheric field lines. Although most studies to date analyze the phase difference in the $H$-component, we find that the phase difference was in fact largest in the $\boldsymbol{Z}$-component. In order to interpret such difference quantitatively, we apply the conventional field line resonance theory to the condition in which the ground signals are induced by the Hall currents in the ionosphere. We also demonstrated that the same formulation can be used to understand the characteristics of field line resonance from the observations of phase differences.
\end{abstract}

(Key words: Magnetic pulsations, ULF waves, Inversion method, Gradient method)

\section{INTRODUCTION}

Ground magnetometers have provided important knowledge of magnetic pulsations (or

\footnotetext{
${ }^{1}$ Institute of Geophysics and Planetary Physics, University of California, Los Angeles, USA

* Corresponding author address: Dr. Peter Chi, Institute of Geophysics and Planetary Physics, 3845

Slichter Hall, 405 Hilgard Ave, Los Angeles, CA 90095-1567 USA; E-mail: pchi@igpp.ucla.edu
} 
ULF waves, with wave periods ranging roughly from $1 \mathrm{sec}$ to $10 \mathrm{~min}$ ) in the magnetosphere for over one hundred years. These ground stations in some aspects are more desirable than satellites in observing magnetic pulsations. In particular, the high travel speeds of satellites at low altitudes make proper observations of low-frequency ULF waves at low $L$-shells impossible. It is also more economical to build a large ground magnetometer array that provides measurements at multiple locations. The cost of such ground systems is usually a small fraction of what is required by a single spacecraft mission.

While several research groups are expanding their magnetometer arrays as well as incorporating modern technologies in the instruments, new and exciting ways to make use of the wealth of information provided by these ground observations have also been perceived in recent years. Two of these techniques, namely the inversion method and the gradient method, are the subject of this paper. The uniqueness of these two techniques is that the analysis requires the input from the data of multiple stations at one time, whereas the traditional way to study multi-station observations repeats the same analysis for each station. Improving these new techniques and applying them to observations are potentially important in providing new insights of the physics of magnetic pulsations.

The first technique is an inversion method to estimate the several factors that affect the wave amplitude observed on the ground (Chi et al. 1996). These factors are the magnitude of the wave event, the local time, and the ground conductivity, all of which can be modeled and estimated through an inversion algorithm. The input data are the wave amplitude values observed by stations located at the same $L$-value but at different local times. This technique is particularly valuable in evaluating the ground conductivity effect that is usually an unknown factor in the analysis of ground pulsations.

After the initial success in using only one day of pulsation data from the five Air Force Geophysics Laboratory (AFGL) stations located at $55^{\circ}$ corrected geomagnetic latitude (Chi et al. 1996), the inversion technique has not been tested by other datasets. In this study we assessed the technique by using 4 months of AFGL data. Having this larger database, we discovered a simpler yet effective way to estimate the conductivity effect, which help obtain the solutions of other factors. We also found that the results were consistent with what were reported by Chi et al. (1996).

The second technique is the gradient method first proposed by Baransky et al. (1985) and later successfully promoted by Waters et al. (1991). It compares the wave signals at two magnetometer stations closely separated in the north-south direction to identify the resonant frequencies of the field line located midway between the two stations. It is a good use of the field line resonance theory that predicts a peak in wave amplitude at the resonance point and a sharp transition of wave phase across the resonance (e.g., Chen and Hasegawa 1974). The gradient method has two different versions: the cross-phase technique subtracts the wave phases observed at the two stations, and the amplitude-ratio technique compares the two wave amplitudes. The cross-phase technique is easier to demonstrate and thus shown more frequently in literature, though it requires synchronized observations at the two stations in order to determine the phases accurately. The amplitude-ratio technique does not have such strict requirement in timing accuracy (Russell et al. 1999), but a calibration of zero level is needed when the ground conductivity at two stations differs (Green et al. 1993). The gradient technique can be used as 
a "remote sensing" of the plasma density in the magnetosphere. Specifically, it has been used to study the depletion and replenishment of the plasmasphere during magnetic storms (e.g., Chi et al. 2000).

Most observational studies exercising the gradient method have been using the conventional field line resonance theory in the magnetosphere to interpret their results. A qualitative description incorporating the well-known phase change across the resonance point and the ionospheric rotation (Hughes and Southwood 1976) has satisfactorily explained the phase difference in the $H$-component in terms of a toroidal mode resonance. However, we recently discovered that the larger phase difference in the Z-component is not immediately answered by the above straightforward interpretation. A quantitative assessment according to existing theories should be made to see if it is consistent with the observations in all three components.

The organization of this paper is as follows: Section 2 presents the characteristics of equilatitude pulsation data and the implications on the inversion method. Section 3 demonstrates the gradient method using equilongitude observations and relevant model calculation. Some possible future developments of these two methods are discussed in Section 4.

\section{THE INVERSION METHOD FOR ANALYZING EQUILATITUDE PULSATIONS}

The rationale of this method is based on the high coherency in wave power modulation across the magnetometer arrays at mid and low latitudes (Chi et al. 1994, 1996). At these latitudes, broadband Pc 3-4 waves are usually found in daytime. Although the cause of detailed wave-power modulation is not clear, there is evidence that the ultimate energy source is the upstream waves in the foreshock region (Chi et al. 1994). The wave amplitude averaged over a time interval of 5-10 min (or an "event") computed by Fourier transform is used in the calculation because the waveform of signals is not necessarily coherent among different locations. For this reason, an accurate synchronization of magnetometer systems is not absolutely required.

The original inversion model considers the wave amplitude observed on the ground $(A)$ as a function of the magnitude of the wave event $(B)$, the local time $(t)$, and the amplification of wave amplitude due to ground conductivity $(\sigma)$ (Chi et al. 1996). Their relationship can be written as

$$
A=B f(t) \sigma,
$$

where the local time dependence function $f(t)$ can be conveniently expressed by a polynomial function

$$
f(t)=1+\sum_{k=1}^{K} a_{k}(t-12)^{k} .
$$

This function is defined as above so $f=1$ at local noon. If there are $N_{s}$ stations located on a same latitude and $N_{e}$ wave events for each station, the quantity

$$
S=\sum_{i=1}^{N_{e}} \sum_{j=1}^{N_{s}}\left(A_{i j}-B_{i} f\left(t_{i j}\right) \sigma_{j}\right)^{2},
$$


is expected to be minimal. We may estimate the values of the defined parameters by minimizing $S$ and solving the following set of nonlinear equations:

$$
\begin{aligned}
& \frac{\partial S}{\partial B_{i}}=0, \quad i=1,2, \cdots N_{e} \\
& \frac{\partial S}{\partial \sigma_{j}}=0, \quad j=1,2, \cdots N_{s}-1 . \\
& \frac{\partial S}{\partial a_{k}}=0, \quad k=1,2, \cdots K .
\end{aligned}
$$

Note that there are only $N_{s}-1$ independent variables for $\sigma$. One can define $\sigma_{N s} \equiv 1$ and obtain the "relative ground conductivity effect" for the other stations. In reality, the equation set (4) has to be solve numerically. However, there are $N_{e}+N_{s}+K-1$ unknowns in those equations, and the numerical calculation can be time-consuming when the number of events $\left(N_{e}\right)$ becomes a large number. The solution to this problem is to replace $B$ by

$$
B_{i}^{*}=\frac{\sum_{j=1}^{N_{s}} \sigma_{j} f\left(t_{i j}\right) A_{i j}}{\sum_{j=1}^{N_{s}}\left(\sigma_{j} f\left(t_{i j}\right)\right)^{2}},
$$

since $\partial S / \partial B_{i}=0$. Therefore, the number of parameters in optimization becomes a fixed number, $N_{s}+K-1$, no matter how many events there are.

In this study, we present a different approach to estimate the unknown parameters without solving the aforementioned optimization problem. This alternative method requires a much larger amount of data for reliable statistics. We collected 440 Ultra-Low-Frequency (ULF) wave events between August and November 1978 from the 5 northern AFGL stations. The locations of these stations are listed in Table 1. The local-time span of these stations is about 4 hours. In order to analyze the results in different wave frequencies, the wave events are divided into three subgroups for $7-15 \mathrm{mHz}, 15-30 \mathrm{mHz}$, and $30-50 \mathrm{mHz}$ bands.

For each local time $t$, we selected the median of the wave amplitude values within the $(t \pm$ 1) bin. It is reasonable to assume that this median wave amplitude is independent of $B$ because the same distribution of $B$ can be expected at every observer's local time. Therefore, the me-

Table 1. Locations of the five northern AFGL stations in corrected geomagnetic coordinates.

\begin{tabular}{|l|r|r|}
\multicolumn{1}{|c|}{ Station } & Latitude & \multicolumn{1}{c|}{ Longitude } \\
\hline Newport (NEW) & $55.2^{\circ}$ & $299.6^{\circ}$ \\
\hline Rapid City (RPC) & $54.1^{\circ}$ & $317.3^{\circ}$ \\
\hline Camp Douglas (CDS) & $56.3^{\circ}$ & $334.2^{\circ}$ \\
\hline Mt. Clemens (MCL) & $55.8^{\circ}$ & $344.8^{\circ}$ \\
\hline Sudbury (SUB) & $55.8^{\circ}$ & $1.9^{\circ}$ \\
\hline
\end{tabular}


dian wave amplitude depends only on the local time and the ground conductivity effect. Figure 1 shows these median wave amplitude for the $15-30 \mathrm{mHz}$ band at different local times. It can be seen that some stations (e.g., MCL) always observed stronger waves.

If we arbitrarily assign that $B=1$, we know from (1) that $\sigma_{j}=A\left(t_{i j}\right) / f\left(t_{i j}\right)$. Since all the five stations have data for $t=8,9, \ldots 15$, we can estimate $\sigma_{j}$ for each local time. The estimated values of $\sigma_{j}{ }^{*}$ average over all local times are plotted in Fig. 2, where $\sigma_{S U B}{ }^{*} \equiv 1$. The values in Fig. 2 are consistent with the results in Chi et al. (1996). When the $\sigma^{*}$ for each station is known, the wave amplitude can be calibrated as $\tilde{A}_{i j} \equiv A_{i j} / \sigma_{j}^{*}$, which represents the expected wave amplitude on the ground if there is no difference in ground conductivity among stations.

The estimated local time dependence function $f^{\prime}(t)$ can be calculated by the median value of $\tilde{A}_{i j}$ within a 2-hour bin centered at $t$ for each local time $t$. Figure 3 shows the $f^{\prime}(t)$ for the pulsation amplitude in three different frequency bands. For the lowest frequency band 7-15 $\mathrm{mHz}$, the peak of $f^{\prime}(t)$ is located in the afternoon for the $H$-component but in the morning for the $D$-component. Although this distinction has been reported by Bloom and Singer (1995) who analyzed two years of AFGL data, we are able to obtain the same result with a much smaller amount of data by using the inversion model (1).

\section{THE GRADIENT METHOD FOR ANALYZING EQUILONGITUDE PULSATIONS}

The gradient method has been a powerful tool to identify the eigenfrequencies of magnetospheric field lines. Before this technique was invented, it was difficult to distinguish the resonant wave power from the driving wave power in the wave spectrum from a single station. The comparison of wave power or phase at two stations closely separated in the north-south direction can effectively screen the driving wave power, and several authors have demon-

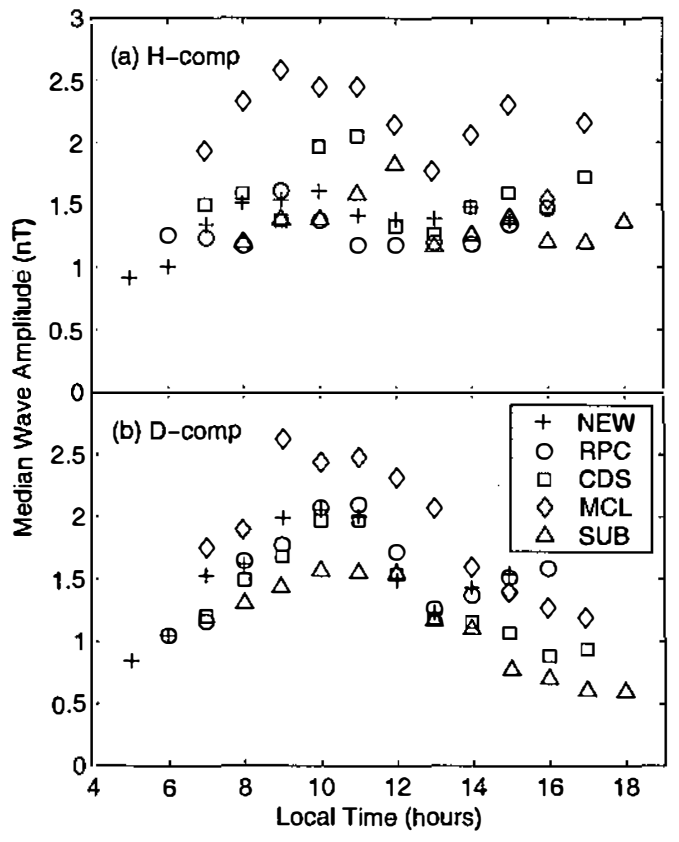

Fig. 1. Median values of the wave amplitudes within 2-hour bins in the $15-30 \mathrm{mHz}$ frequency band. Data are selected from 440 wave events between $\mathrm{Au}$ gust and November in 1978. 


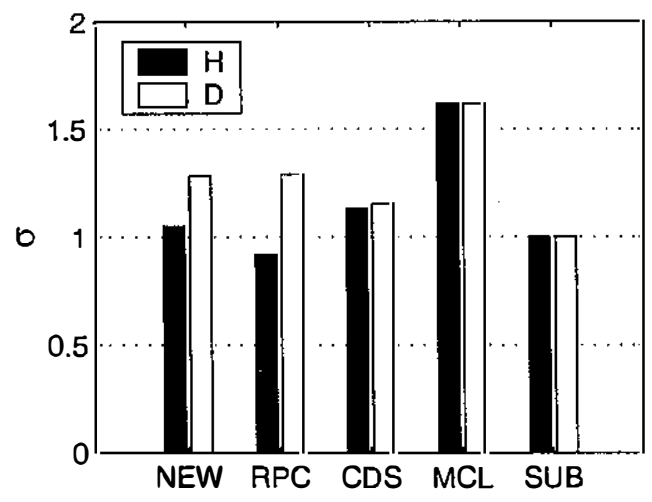

Fig. 2. Amplification factors in the $H$ - and $D$ components for the five northern AFGL stations. The $\sigma$ for Sudbury (SUB) is set to be 1 for both components.

strated successful cross-phase results using station pairs with baselines of roughly $100 \mathrm{~km}$ (Best et al. 1986; Waters et al. 1991; Green et al. 1993).

A demonstration of the cross-phase technique is presented in Fig. 4. The data were taken at two British magnetometer stations, Durness (DU) and Loch Laggan (LL), located along the $60^{\circ}$ geomagnetic meridian where the local time is roughly the same as Universal Time (UT). The geomagnetic latitudes of Durness and Loch Laggan are $61.46^{\circ}$ and $59.89^{\circ}$, respectively, and the two stations are separated by approximately $184 \mathrm{~km}$ in the north-south direction. Figure 4a is the spectrogram of the magnetic field measured at Loch Laggan on May 9, 1979.
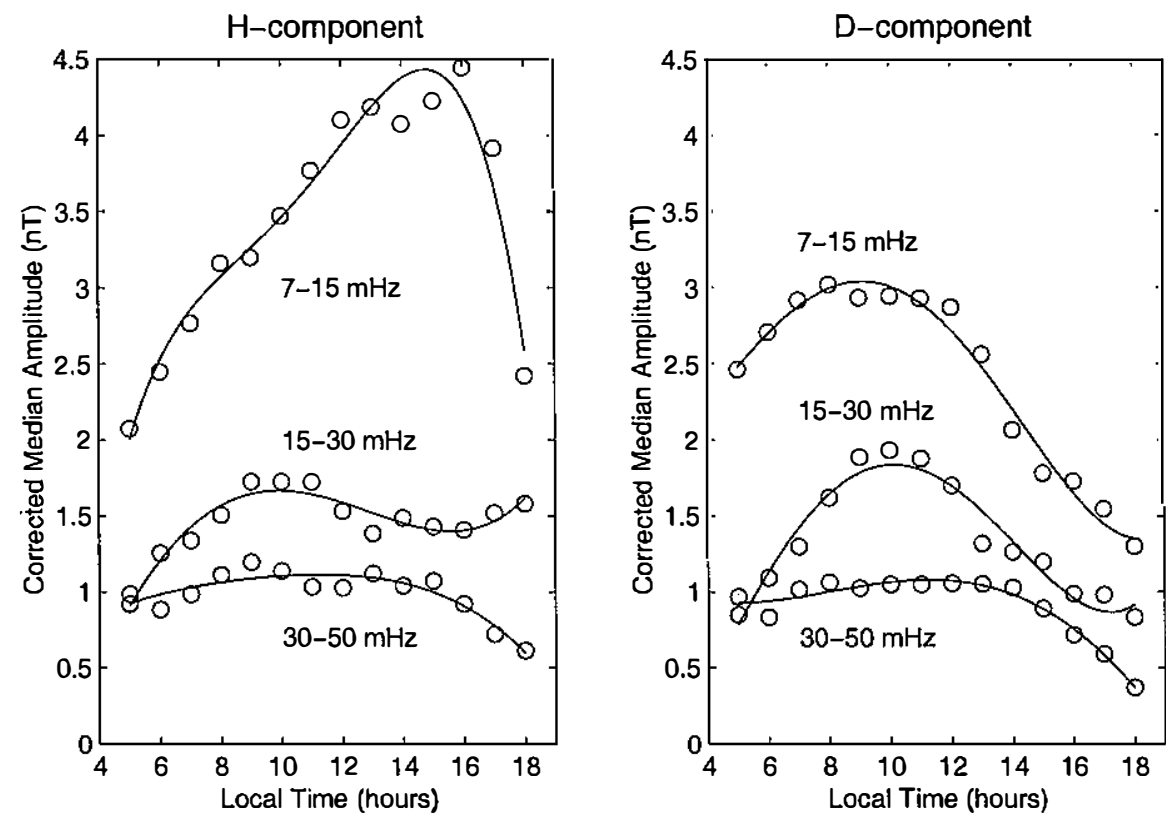

Fig. 3. (Left) Calibrated median wave amplitudes in the $H$-component that represent the local time dependence function $f(t)$ for three different frequency bands. The solid lines are the best fourth-order polynomial fits. (Right) Same except for the $D$-component. 
(a) Spectral Density of $B_{\mathrm{LL}}$ ( $\mathrm{H}$ component)

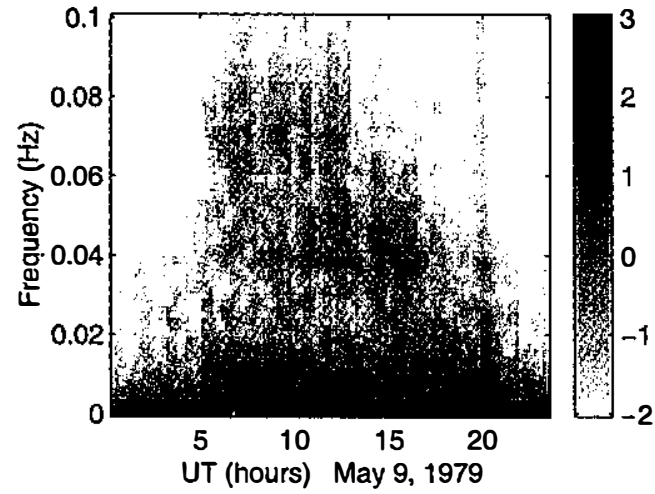

(c) $\phi_{L L}-\phi_{D U}$ (D component)

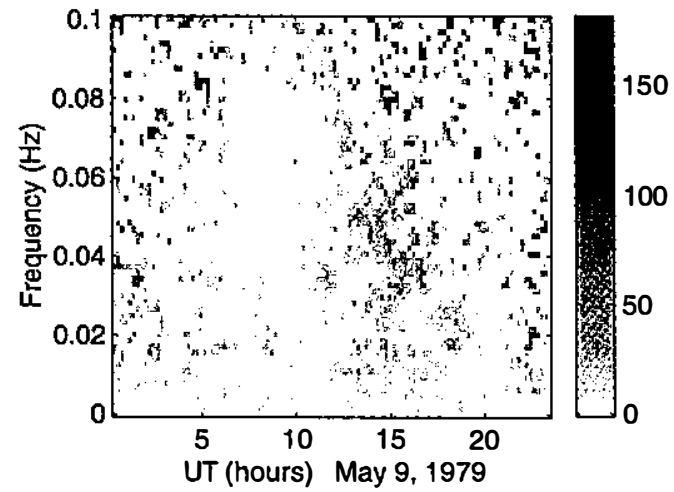

(b) $\phi_{L L}-\phi_{D U}$ (H component)

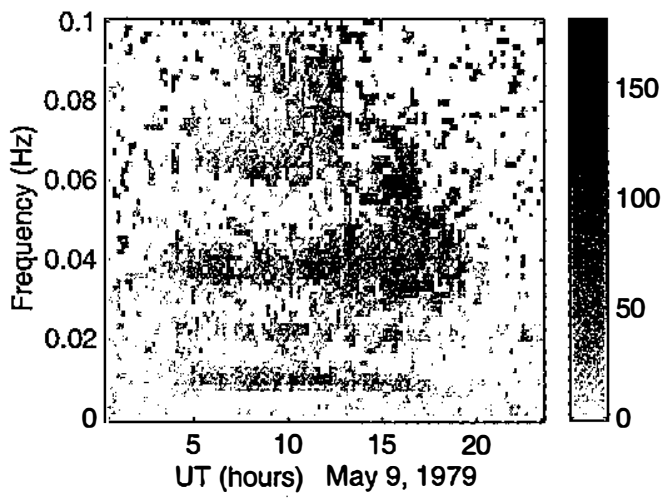

(d) $\phi_{L L}-\phi_{D U}$ (Z component)

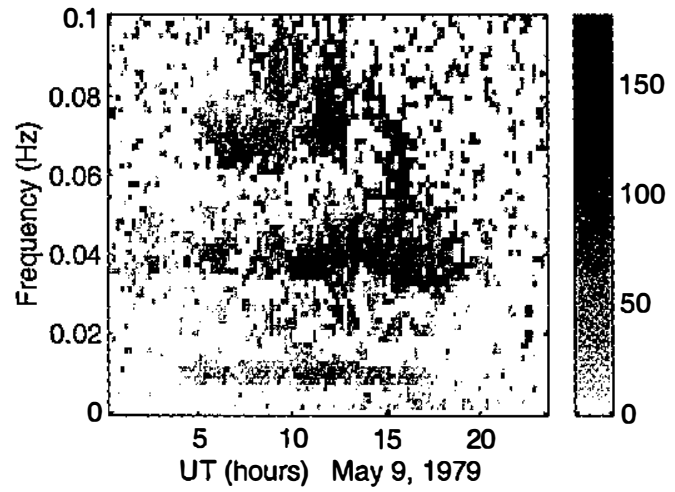

Fig. 4. (a) Power spectrogram of BH observed at Loch Laggan. (b),(c),(d) Crossphase spectrograms for the Durness-Loch Laggan magnetometer pair in the $H$-component, $D$-component, and $Z$-component, respectively.

Enhanced wave power in the Pc 3-4 band was observed during the daytime over the entire Pc 3-4 band. A similar wave activity was also found at Durness.

The cross-phase spectrum is the phase difference between the two time series expressed as a function of time and frequency. Figures $4 \mathrm{~b}-4 \mathrm{~d}$ are the cross-phase spectrograms for the $H$ (northward), $D$ (eastward), and $Z$ (downward) components of the Durness-Loch Laggan pair on May 9, 1979. The phase difference is meaningful when the two signals are coherent. When the wave power is weak and the coherency is low, the values of phase difference can sometimes appear in an expected random fashion. We found that plotting the values only when the coherency is larger than 0.3 reduces most of the randomness, and we adopted such a criterion when making Figs. 4b-4d. In addition, since the phase difference $\phi_{L L}-\phi_{D U}$ is basically larger than 0 , only positive values are shown. For the $H$ component (Fig. 4b), horizontal patterns of enhanced phase differences are seen at approximately $10,22,38$, and $66 \mathrm{mHz}$ in the daytime. The same patterns with even greater phase differences are seen in the $Z$-component. These patterns are associated with the harmonics of field line resonance in the magnetosphere, and 
the corresponding frequencies are the resonant frequencies for the field lines between the latitudes of the two stations (Chi and Russell 1998). Note that the above harmonics cannot be easily identified in the power spectrogram (Fig. 4a). Showing a clear contrast, the phase difference in the $D$-component (Fig. 4c) is much smaller and there exists no discernible pattern.

It is worthwhile to clarify the physics behind of the observations of phase differences as presented above. In addition to the conventional field line resonance theory (Southwood 1974; Chen and Hasegawa 1974), a simpler two-oscillator model that can interpret the phase-difference observations has also been proposed (Waters et al. 1994). The two-oscillator model considers the two field lines that connect to the two ground stations 1 and 2, where 1 denotes the station at higher latitude. The assumption is that the signals seen on the ground stations are caused only by the oscillations of the corresponding field lines driven by a common source. Waters et al. (1994) further found that the phase difference $\phi_{2}-\phi_{1}$ maximized at $\omega=\left(\omega_{1}+\omega_{2}\right) / 2$, where $\omega_{1}$ and $\omega_{2}$ are the eigenfrequencies of the two field lines. However, Chi and Russell (1998) pointed out that there is an intrinsic difference in physics between the field line resonance theory and the two-oscillator model. The field line resonance theory models the three-dimensional plasma in the magnetosphere and finds how the system responds to a common driving wave. The theory predicts only one resonance where the phase encounters a sharp transition at the resonant frequency. A proper interpretation of the phase-difference observations on the ground should include the field line resonance theory in the magnetosphere and the induction due to the associated ionospheric currents.

To interpret the observations in Fig. 4, we began our calculation with the "box model" (Southwood 1974), which assumes a homogeneous magnetic field in the $z$ direction. The plasma density is assumed to be inhomogeneous in the $x$ or outward direction to account for the varying Alfvén velocity in the realistic magnetosphere. The $y$ direction is determined by $\mathbf{y}=\mathbf{z} \times \mathbf{x}$. A surface wave on the magnetopause with a wavenumber $\lambda$ in the $y$ direction can propagate evanescently inward and result in a resonance where the frequency matches an eigenfrequency of the field line. The resonance can be expressed by the following equation (Southwood 1974):

$$
\frac{d^{2} E_{y}}{d x^{2}}+\frac{1}{x-x_{0}-i \varepsilon} \frac{d E_{y}}{d x}-\lambda^{2} E_{y}=0,
$$

where $\varepsilon$ is a small quantity due to dissipation and representative of the resonance width. The solution of (6) is

$$
E_{y}(x)=C I_{0}\left(\lambda\left(x-x_{0}+i \varepsilon\right)\right)+D K_{0}\left(\lambda\left(x-x_{0}+i \varepsilon\right)\right),
$$

where $I_{0}$ and $K_{0}$ are modified Bessel functions. The electric field in the $x$ direction is given by

$$
E_{x}=-\frac{i \lambda}{\left(\omega / v_{A}\right)^{2}-k^{2}-\lambda^{2}} \frac{d E_{y}}{d x},
$$

where $v_{A}$ is the Alfvén velocity and $k$ is the wavenumber in the $z$ direction.

Figure 5 depicts the amplitudes and phases of $E_{x}$ and $E_{y}$ solved from (7) and (8) when appropriate boundary conditions are given. The wavenumber $\lambda$ is set to be $1 R_{E}$. The resonant location $x_{0}$ is taken to be 0 . It is clear that both $E_{x}$ and $E_{y}$ maximize at the resonant point with different finite magnitudes. The fact that $\left|E_{x}\right|$ is much greater than $\left|E_{y}\right|$ indicates that the 

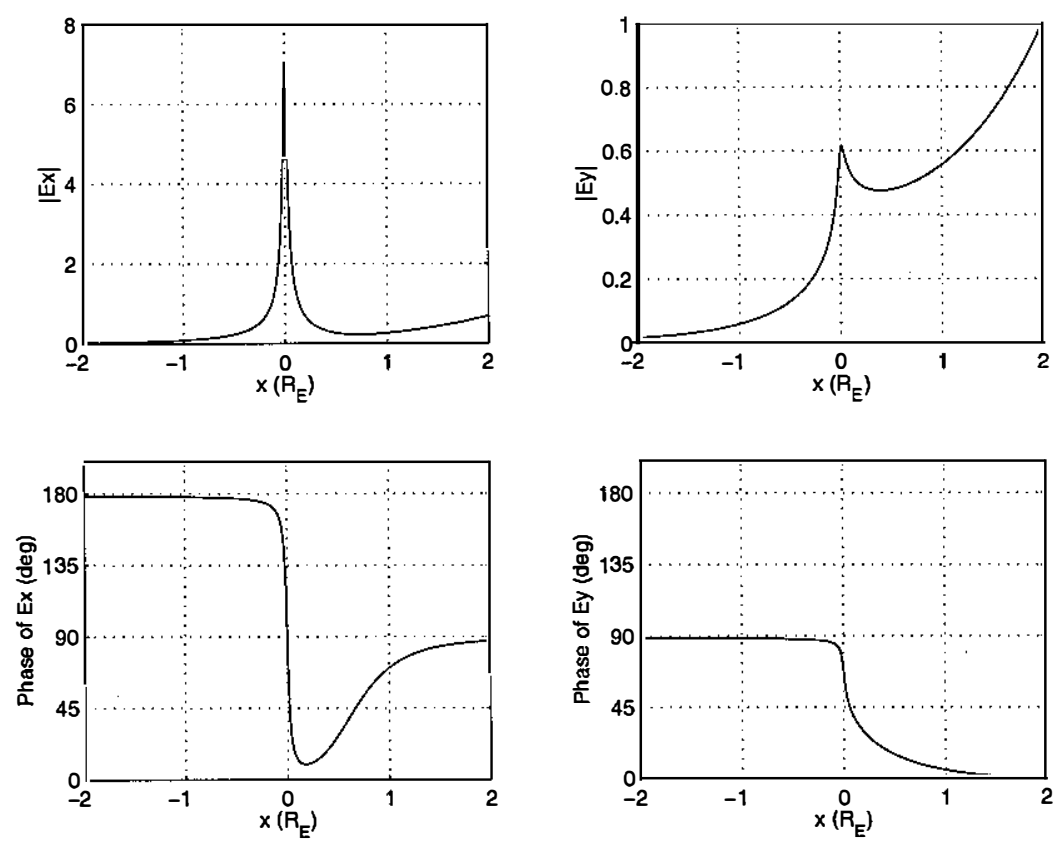

Fig. 5. Amplitude and phase of $E_{x}$ and $E_{y}$ as functions of $x$.

electric oscillations are mainly in the radial direction. The phases of both $E_{x}$ and $E_{y}$ change dramatically at the resonant point, where the phase change in $E_{x}$ is roughly $180^{\circ}$.

When $E_{x}$ and $E_{y}$ are given, the Pedersen and Hall currents in the ionosphere can be written as

$$
\left(\begin{array}{l}
J_{x} \\
J_{y}
\end{array}\right)=\left(\begin{array}{ll}
\sum_{P} & \sum_{H} \\
-\sum_{H} & \sum_{P}
\end{array}\right)\left(\begin{array}{l}
E_{x} \\
E_{y}
\end{array}\right),
$$

where $\sum_{P}$ and $\sum_{H}$ are height-integrated Pedersen and Hall conductivities, respectively. Since the effect of the Pedersen currents cancels the incident wave, the magnetic oscillations on the ground only come from the contribution of the Hall currents. Figure 6 shows the four phases of Hall currents in one wave period. The Hall-current oscillations are mainly in the $y$ direction because $E_{x}$ dominates the electric field.

The ground magnetic signals induced by the ionospheric Hall currents can be calculated by using the Biot-Savart law, and the results are presented in Fig. 7. Two cases for different resonant widths $(\varepsilon)$ are shown. The two plots on the left show the wave amplitude and the phase when $\varepsilon=h$, where $h$ is the ionospheric height and is approximately $110 \mathrm{~km}$ in reality. The horizontal axis $x$ is the displacement in magnetic latitude in a unit of $h$, and the vertical axis $|b|$ is in a relative unit so that the wave amplitude at $x=0$ in the $H$-component is 1 . The results in Fig. 7 indicate that the $|b|$ in the $H$ - and $Z$-components are comparable, and the $|b|$ in the $D$ component is negligible because the ionospheric Hall currents are mainly in the same direction. It is interesting to note that although most cross-phase observations up to date have 

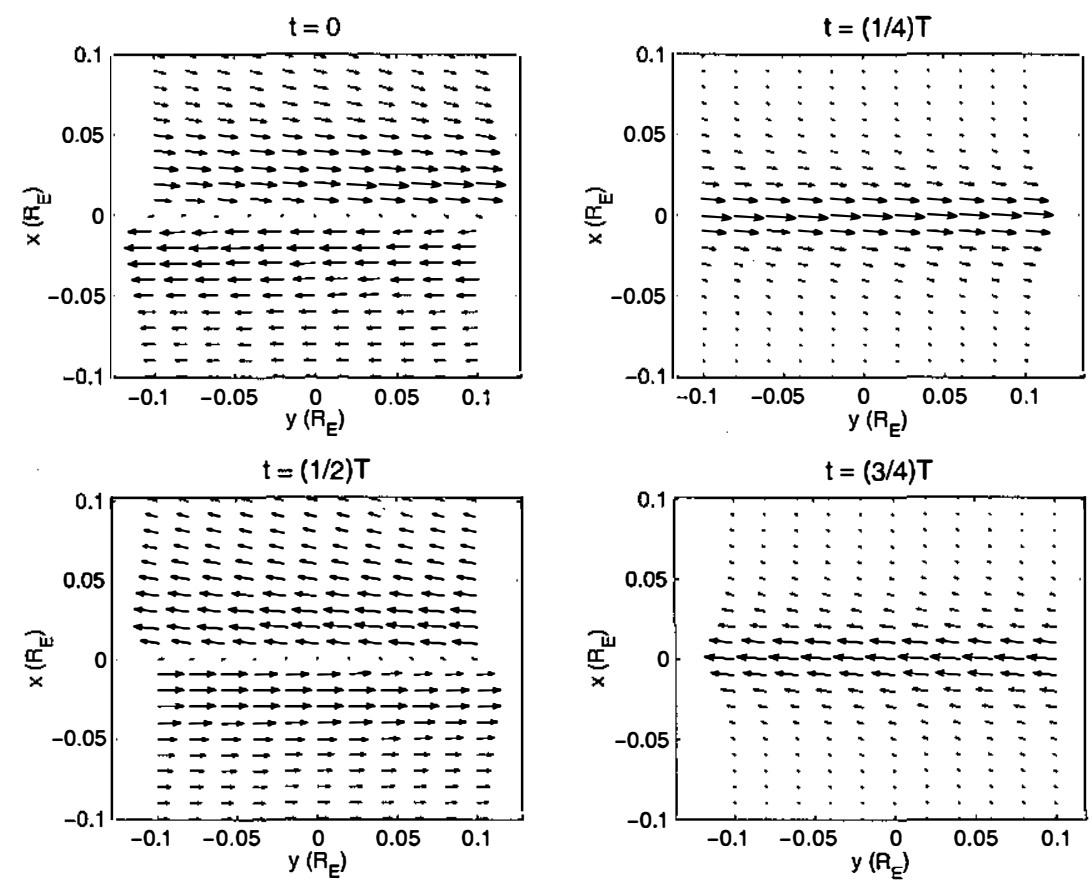

Fig. 6. Ionospheric Hall currents associated with a field line resonance at four different phases in a wave cycle.

been focusing on the $H$-component, the phase change across the resonance is in fact the greatest in the $Z$ component. The phase variation in the $D$-component is rather negligible. The two plots on the right show the same calculation but for the condition when $\varepsilon=5 h$. In this case the wave amplitude is noticeably smaller than that in the previous case due to a weaker resonance that covers a wider latitudinal span. Different from the previous case, the wave amplitude in the $Z$ component is now clearly smaller than that in the $H$-component because the $Z$-component is induced by the strong opposite currents at neighboring latitudes, which are farther from the observer in this case. The phase change is also more gradual than in the previous case due to the wider resonance width.

The above calculation can be easily expanded to include the effect of ground conductivity by adding the induction due to the image current located inside the Earth. This electrostatic approach is valid for the mid- and low-frequency ULF waves that are relevant here. For example, if an ionospheric current $d \mathbf{I}$ is located at $z=h$ and the Earth's conductivity is $\sigma$, the associated image current $-d \mathbf{I}$ is flowing underground at $z=-(h+2 p)$ where $p$ is the skin depth $\left(\mu_{0} \omega \sigma\right)^{-1}$. Therefore, the total magnetic field on the ground is

$$
\mathbf{B}=\int \frac{\mu_{0}}{4 \pi}\left(\frac{d \mathbf{I} \times \mathbf{R}_{1}}{R_{1}^{3}}-\frac{d \mathbf{I} \times \mathbf{R}_{2}}{R_{2}^{3}}\right),
$$

where $\mathbf{R}_{\mathbf{1}}$ and $\mathbf{R}_{\mathbf{2}}$ are the vectors from the ionospheric current and the image current, respectively, 

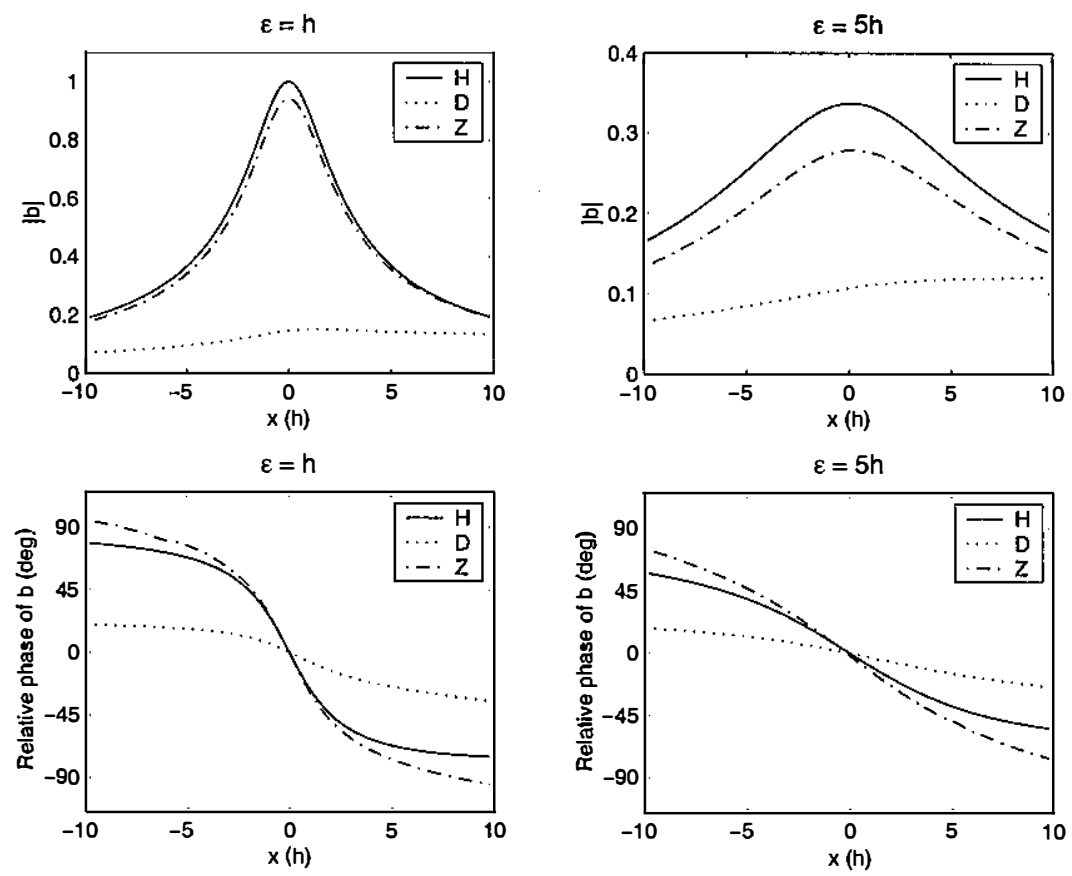

Fig. 7. Wave amplitude and phase of the magnetic field oscillations on the ground due to a field line resonance. (Left) When $\varepsilon=h$. (Right) When $\varepsilon=5 h$.

to the ground observer. When the Earth's conductivity $\sigma$ is $0, p$ is infinitely large, and consequently only the first term in (10) exists. On the other hand, when the Earth is perfectly conductive, $p=0$ and the magnetic field is twice as strong as that for the zero-conductivity case if there is no lateral distance between the currents and the ground observer (since $\mathbf{R}_{2}=-\mathbf{R}_{1}$ ). This approach is similar to the analysis of geomagnetically induced currents (e.g., Pirjola and Viljanen 1998), which are an important manifestation of space weather in modern technologies such as the transformers in power systems.

\section{LOOKING AHEAD}

We have presented some recent progress in understanding and interpreting the results of the inversion method and the gradient method. For the inversion method, we introduced an alternative approach to analyze the same inversion problem without performing inversion. We analyzed four months of pulsation amplitude data and confirmed the earlier results of ground conductivity effect as found in Chi et al. (1996). For the gradient method, we illustrated the essential pieces in the theory of field line resonance that are relevant to interpreting the results of the gradient method. We added the ionospheric effect to demonstrate the different results in the three magnetic field components. Our calculation shown in Fig. 7 also indicates that the characteristics of field line resonance can be studied by the gradient method.

It is expected that both methods will stimulate more research and provide further insights 
of the physics of magnetic pulsations for years to come. The immediate use of the inverse method is to calibrate the wave amplitude observed on the ground. The gradient method has been used to estimate the plasma density inferred from eigenfrequencies as a tool to monitor space weather. Besides these practical purposes, the two methods are potentially important in advancing our understanding of the different resonances of the magnetosphere as well as the magnetosphere-ionosphere-atmosphere-ground system in which magnetic pulsations take place. The following are our views of the possible areas where these two methods could bring further impacts.

\subsection{The Inversion Method}

The products of the inversion method can be used to investigate the source of magnetic pulsations. For example, the local time dependence function $f(t)$ is useful in staying the geometry of source energy and the ionospheric effect on pulsation amplitude. The magnitude of wave events $B$ can be correlated with other physical phenomena to find the preferred conditions for magnetic pulsations to occur. This is analogous to the methodology comparing the $K p$ index with solar wind parameters to infer the causes of geomagnetic disturbances. In fact, $B$ is a better quantity than $K p$ for such purpose because it is decoupled from the local time dependence and the ground conductivity effect. It might be useful to generate the "calibrated $K p$ index" in a similar way.

Both the physical model and the optimization formulation of the inversion method may need further modifications. For the model part, one could consider $\sigma$ as a tensor. Since the ground conductivity structure can be inhomogeneous, it is possible that the current flowing in the $x$ direction in the ionosphere will induce magnetic field in both $y$ and $x$ directions on the ground. However, the inclusion of the off-diagonal terms in the $\sigma$ tensor will increase the number of parameters in the model, and it is expected that the reliability of estimation will be reduced.

The optimal number of parameters is also an important issue in the optimization problem. In the original model (Chi et al. 1996), the local time dependence function $f(t)$ is modeled by a fourth-order polynomial. A different order of polynomial or different functional forms are also possible choices, but a model selection criterion such as the Akaike Information Criterion (Akaike 1974) can be used to determine the best model. The same approach can also be applied to assess if a tensor form for $\sigma$ is appropriate. The ground conductivity structure for some stations may be homogeneous, and therefore the associated $\sigma$ can be vectors rather than tensors in the model.

A possible long-term goal is to include the magnetotelluric (MT) method in geophysical exploration to obtain the detailed knowledge of ground conductivity. The $\sigma$ parameter can be known from the MT method, and the number of parameters to be optimized in the inversion model will be reduced. However, the MT method requires a high-density array of magnetometers in a relatively smaller region, whereas the magnetometers for studying space physics phenomena need to be more dispersive in order to cover a much larger area. Some experiments coordinated by geophysicists and space physicists may resolve this difficulty and benefit both fields. 


\subsection{The Gradient Method}

The comparison between the two-oscillator model proposed by Waters et al. (1994) and the conventional field line resonance model immediately raises the question about the number of resonant $L$-shells in the magnetosphere. The two-oscillator model assumes that the two field lines connecting to the station pair are resonating. If we have an infinite amount of magnetometer stations, the two-oscillator model implies an unrealistic situation where an infinite amount of resonant $L$-shells exist. The conventional field line resonance model discusses the condition to the other extreme. The model consists of one driving wave and a single resonance in the magnetosphere where the driving frequency matches a resonant frequency of the field line. The resonance has a width that is associated with the dissipation of wave energy in the ionosphere. A convincing piece of observational evidence for such single-resonance case has been provided by Walker et al. (1979), who found in radar observations that the Pc 5 waves in the outer magnetosphere had a resonance width of approximately $1^{\circ}$ in latitude. It can be expected that multiple resonances can exist in the magnetosphere by superposition, but the field line resonance theory does not go further to predict how many resonant $L$-shells there can be.

The cavity mode model proposed by (Kivelson and Southwood 1985) provides a solution to this problem. In their model, field lines resonate only when the field line resonance frequency coincides with the cavity mode frequency, so the number of resonances is finite. Therefore, not all the harmonics of a field line may be seen at single latitude if the frequency separation of two consecutive cavity modes is wide enough. It may be possible to find the evidence for the above prediction by observing the cross-phase spectrum from a chain of magnetometers on the same longitude.

So far the gradient method has only considered the magnetometer stations located along the north-south direction. Performing a similar analysis with an east-west magnetometer pair to examine the azimuthal structure of field line resonances may also generate intriguing results. Except for the structure of small azimuthal wavelengths that cannot be seen on the ground due to the ionospheric screening effect (Hughes and Southwood 1976), the phase differences in the east-west direction can tell us the azimuthal wave number. In the ideal condition as described in the conventional field line resonance theory, the signals at two stations separated in the east-west direction are always coherent regardless of the distance between them. However, this may not be true in reality, and the information about the azimuthal coherence may hint to us the energy sources of resonances. Expanding the gradient method from one dimension to two dimensions may be a trend as more two-dimensional magnetometer arrays develop.

Acknowledgments P. J. Chi is indebted to T. Higuchi of the Institute of Statistical Mathematics in Japan for developing the optimization algorithm of the inversion method.

\section{REFERENCES}

Akaike, H., 1974: A new look at the statistical model identification. IEEE Trans. Automatic Control, AC-19, 716-723. 
Baransky, L. N., J. E. Borovkov, M. B. Gokhberg, S. M. Krylov, and V. A. Troitskaya, 1985: High resolution method of direct measurement of the magnetic field lines' eigenfrequencies. Planet. Space Sci., 33, 1396-1374.

Best, A., S. M. Krylov, Yu. P. Kurchashov, Ya. S. Nikomarov, and V. A. Pilipenko, 1986: Gradient-time analysis of Pc 3 pulsations. Geomagn. and Aeronomy, 26, 829-832.

Bloom, R. M., and H. J. Singer, 1995: Diurnal trends in geomagnetic noise power in the Pc 2 through Pc 5 bands at low geomagnetic latitudes. J. Geophys. Res., 100, 14943-14953.

Chen, L., and A. Hasegawa, 1974: A theory of long-period magnetic pulsations, 1. Steady state excitation of field line resonance. J. Geophys. Res., 79, 1024-1032.

Chi, P. J., and C. T. Russell, 1998: An interpretation of the cross-phase spectrum of geomagnetic pulsations by the field line resonance theory. Geophys. Res. Lett., 25, 4445-4448.

Chi, P. J., C. T. Russell, and G. Le, 1994: Pc 3 and Pc 4 activity during a long period of low interplanetary magnetic field cone angle as detected across the Institute of Geological Sciences array. J. Geophys. Res., 99, 11127-11139.

Chi, P. J., C. T. Russell, G. Le, W. J. Hughes, and H. J. Singer, 1996: A synoptic study of Pc 3,4 waves using the Air Force Geophysics Laboratory magnetometer array. $J$. Geophys. Res., 101, 13215-13224.

Chi, P. J., C. T. Russell, S. Musman, W. K. Peterson, G. Le, V. Angelopoulos, G. D. Reeves, M. B. Moldwin, and F. K. Chun, 2000: Plasmaspheric depletion and refilling associated with the September 25, 1998 magnetic storm observed by ground magnetometers at $L=2$. Geophys. Res. Lett., 27, 633-636.

Green, A. W., E. W. Worthington, L. N. Baransky, E. N. Fedorov, N. A. Kurneva, V. A. Pilipenko, D. N. Shvetzov, A. A. Bektemirov, and G. V. Philipov, 1993. Alfven field line resonances at low latitudes $(L=1.5)$. J. Geophys. Res., 98, 15693-15699.

Hughes, W. J., and D. J. Southwood, 1976: The screening of micropulsation signals by the atmosphere and ionosphere. J. Geophys. Res., 81, 3234-3240.

Kivelson, M. G., and D. J. Southwood, 1985: Resonant ULF waves: a new interpretation. Geophys. Res. Lett., 12, 49-52.

Pirjola, R., and A. Viljanen, 1998: Complex image method for calculating electric and magnetic fields produced by an auroral electrojet of finite length. Ann. Geophysicae, 16, 1434-1444.

Russell, C. T., P. J. Chi, V. Angelopoulos, W. Goedecke, F. K. Chun, G. Le, M. B. Moldwin, and E. G. Reeves, 1999: Comparison of three techniques of determining the resonant frequency of geomagnetic pulsations. J. Atmos. Solar-Terr. Phys., 61, 1289-1297.

Southwood, D. J., 1974: Some features of field line resonances in the magnetosphere. Planet. Space Sci., 22, 483-491.

Walker, A. D. M., R. A. Greenwald, W. F. Stuart, and C. A. Green, 1979: Stare auroral radar observations of Pc 5 geomagnetic pulsations. J. Geophys. Res., 84, 3373-3388.

Waters, C. L., F. W. Menk, and B. J. Fraser, 1991: The resonance structure of low latitude Pc3 geomagnetic pulsations. Geophys. Res. Lett., 18, 2293-2296.

Waters, C. L., F. W. Menk, and B. J. Fraser, 1994: Low latitude geomagnetic field line resonances: Experiment and modeling. J. Geophys. Res., 99, 17547-17558. 